\title{
Comportamiento frente a la corrosión de fundiciones con grafito laminar y esferoidal parcialmente modificadas con silicio en $\mathrm{NaCl} \mathbf{0 , 0 3} \mathrm{M}$
}

\author{
María Ángeles Arenas ${ }^{\mathrm{a}}$, Andrea Niklas ${ }^{\mathrm{b}}$, Ana Conde $^{\mathrm{a}}$, Susana Méndez \\ Jon Sertucha ${ }^{\mathrm{b}}$, Juan José de Damborenea ${ }^{\mathrm{a}, \bowtie}$ \\ åDepartamento de Ingeniería de Superficies, Corrosión y Durabilidad. Centro Nacional de Investigaciones Metalúrgicas (CENIM). \\ CSIC. Avda. Gregorio del Amo 8, 28040 Madrid, España \\ bÁrea de Ingeniería, I + D y Procesos Metalúrgicos, IK4-AZTERLAN, Aliendalde 6, E-48200 Durango (Bizkaia) \\ Autor para la correspondencia: jdambo@cenim.csic.es
}

Enviado: 28 Julio 2014; Aceptado: 29 Septiembre 2014; Publicado on-line: 10 D iciembre 2014

RESUMEN: El uso creciente de fundiciones esferoidales en aplicaciones tecnológicas hace que se busquen nuevos métodos para mejorar su comportamiento frente a la corrosión. Entre las distintas maneras de abordar este problema, la modificación de su composición química tiene un doble interés dado que, junto con un aumento en la resistencia frente a la corrosión, se pueden conseguir propiedades mecánicas mejoradas. Todo ello se debe conseguir, además, sin aumentar de manera significativa los costes de producción. En este trabajo se presentan los primeros resultados del comportamiento frente a la corrosión de una serie de fundiciones grafíticas de composición modificada. En el caso de las fundiciones esferoidales se ha variado el contenido de silicio con el fin de conocer el efecto de este elemento. Los resultados encontrados muestran una ligera mejora en la resistencia frente a la corrosión en aquellas aleaciones con alto contenido de silicio respecto a las fundiciones convencionales durante las primeras horas de ensayo para, posteriormente, mostrar un comportamiento similar. No obstante, es importante destacar que estas modificaciones no comprometen las propiedades mecánicas de las aleaciones base, lo que señala un camino para la preparación de nuevas fundiciones con un mayor contenido en elementos de aleación.

PALABRAS CLAVE: Corrosión; Fundición esferoidal; Técnicas electroquímicas

Citation / Cómo citar este artículo: Arenas, M.A., Niklas, A., Conde, A., Méndez, S., Sertucha, J., de Damborenea, J.J. (2014) "Comportamiento frente a la corrosión de fundiciones con grafito laminar y esferoidal parcialmente modificadas con silicio en NaCl 0,03 M". Rev. Metal. 50(4): e032. doi: http://dx.doi.org/10.3989/revmetalm.032.

ABSTRACT: Corrosion behaviour of ductile cast irons partially modified with silicon in $0.03 \mathrm{M} \mathrm{NaCl}$. The increasing demand of ductile cast irons with extensive technological applications leads to enlarge the corrosion resistance of this group of metallic materials. In this sense, the use of different chemical compositions on such cast irons becomes one of the most interesting aspects among the different ways to improve their behaviour against corrosion due to the extra opportunity for increasing the mechanical properties. Additionally such improvements have to be made without any increase of processing costs to keep the interesting competitiveness of developed cast irons. In the present work the preliminary results obtained from corrosion tests made on a group of cast irons with different chemical compositions are presented. Among ductile cast irons, silicon content has been varied in order to investigate the effect of this element on corrosion resistance of the alloys. The obtained results show a slight improvement of this property for the alloys with high silicon content with respect to the conventional ones though such effect was found in the first time period of the corrosion tests. Interestingly this 
improvement was found for alloys that exhibit better tensile properties than the conventional ductile irons. Thus an important way for developing new ductile cast irons with improved corrosion properties by alloying has been opened.

KEYWORDS: Corrosion; Ductile cast iron; Electrochemical techniques

Copyright: (C) 2014 CSIC. This is an open-access article distributed under the terms of the Creative Commons Attribution-Non Commercial (by-nc) Spain 3.0 License.

\section{INTRODUCCIÓN}

Las fundiciones base hierro son, sin lugar a dudas, uno de los materiales más empleados debido principalmente a sus altas prestaciones mecánicas y bajo coste (Tiedje, 2010). Por esta razón, la mayor parte de los sistemas de conducción y distribución de aguas se han venido haciendo en fundiciones de hierro desde hace más de 500 años. A sus buenas propiedades mecánicas se une su fácil maquinabilidad, lo que permite incorporar sistemas auxiliares en las conducciones sin problemas añadidos. A principios de la década de los 50 del siglo pasado, y como consecuencia directa del reto de la industria en controlar la forma del grafito, se desarrollaron las fundiciones esferoidales, que sumaban a las propiedades de las anteriores un menor peso por metro de conducción, una mayor capacidad de carga por unidad de diámetro y una mayor resistencia a la fractura (DeBerry et al., 1982). Sin embargo, ambas fundiciones presentan una resistencia a la corrosión similar, pudiendo considerarse relativamente baja. A pesar de ello, la excelente relación entre propiedades y precio ha hecho que las fundiciones esferoidales se hayan introducido con fuerza en otras aplicaciones industriales como válvulas, bombas, tubos, etc., donde a las solicitaciones mecánicas se suman ambientes corrosivos e, incluso, temperaturas elevadas (colectores de motor, intercambiadores, etc.).

En general, los fabricantes de piezas de fundición esferoidal sufren importantes inconvenientes relacionados con los procesos de corrosión en las piezas que fabrican, siendo la corrosión atmosférica el principal problema al que se encuentran sometidas las piezas fabricadas en fundición esferoidal. La diversidad de atmósferas en las que deben operar este tipo de piezas hace todavía más difícil predecir su comportamiento ya que éstas pueden ser de baja corrosividad, ambientes rurales principalmente, o en medios en los que el contenido en cloruros es elevado por su proximidad a la costa, todo ello con diferentes grados de humedad (Tullmin and Roberge, 2000). Como resultado de su exposición al ambiente, este tipo de materiales desarrollan sobre su superficie una serie de óxidos e hidróxidos de hierro que la recubren de manera homogénea. Dependiendo de la adherencia de esta capa, se dificultará o acelerará el avance de la corrosión. Morcillo et al. (2011) han publicado recientemente una completa revisión sobre los efectos de la corrosión atmosférica en aceros suaves en la que se presentan de manera detallada los principales mecanismos de la formación de óxidos y la morfología de los mismos. Otro de los problemas que presentan las piezas fabricadas con fundición esferoidal es su almacenamiento previo a las condiciones de operatividad. Éste suele ser en ambientes húmedos, lo que origina un problema incipiente de corrosión que obliga a granallar las piezas almacenadas antes de ser utilizadas, con el consiguiente sobrecoste, así como el riesgo de que no sean eliminados todos los óxidos formados originando problemas posteriores.

Los nuevos parques eólicos son un ejemplo perfecto donde las fundiciones se encuentran con distintos tipos de atmósferas según su ubicación. En este caso, donde se precisa de piezas de gran tamaño, los tiempos de acabado y almacenaje son prolongados y la corrosión superficial sí supone un problema importante durante las etapas de pintado y mecanizado. Por todo ello y dada la necesidad de minimizar los costes de producción en las plantas de fundición europeas, existe una creciente demanda para diseñar métodos que permitan ralentizar la corrosión superficial de las piezas fabricadas. Existen, principalmente, dos maneras de mejorar el comportamiento frente a la corrosión de este tipo de materiales. En primer lugar, la utilización de tratamientos superficiales de protección temporal, tratamientos que deben ser tanto fáciles de aplicar como de eliminar (para facilitar operaciones posteriores como, por ejemplo, pintado). El otro método, más complejo pero en el que se está investigando de manera intensa, es el de la modificación de la composición química del material (Zhou et al., 2007; Camba et al., 2010; Sun et al., 2012), de manera que la relación entre silicio y carbono permita conseguir una mejora en la resistencia frente a la corrosión de estas fundiciones con una ductilidad y resistencia mecánica compatibles con su procesado.

En el presente trabajo se ha estudiado el comportamiento frente a la corrosión de cuatro fundiciones convencionales (esferoidal ferrítica, esferoidal perlítica, esferoidal martensítica y perlítica laminar) y de tres modificadas con contenidos variables de $\mathrm{Si}$ como elemento principal de aleación, con el fin de analizar los cambios en su comportamiento frente a la corrosión electroquímica en medios con cloruros de agresividad moderada. Las fundiciones esferoidales con elevados contenidos de Si muestran una 
matriz mayoritariamente ferrítica, por lo que presentan altas ductilidades y comportamientos favorables frente al mecanizado, aunque dicha matriz se endurece considerablemente, aumentando la carga de rotura y el límite elástico del material (de la Torre et al., 2014).

\section{MATERIALES Y MÉTODOS}

El trabajo desarrollado consistió, en primer lugar, en la fabricación de tres fundiciones esferoidales de microestructura ferrítica, perlítica y martensítica. A partir de cada una de estas aleaciones se obtuvo un par de piezas rectangulares, de dimensiones $190 \times 90 \times 12 \mathrm{~mm}$ y con la composición química indicada en la Tabla 1. En cada caso, el metal fundido se preparó en un horno de inducción de media frecuencia $(250 \mathrm{~Hz})$, con $100 \mathrm{~kW}$ de potencia y capacidad máxima para $100 \mathrm{~kg}$. Las cargas metálicas estaban constituidas por $55 \%$ de lingote y $45 \%$ de retornos procedentes de la fabricación de piezas ferríticas pertenecientes al sector eólico. Tras la fusión de las cargas, se ajustaron los contenidos de $\mathrm{C}$ y Si mediante la adición de grafito de electrodo (\% en masa, $\mathrm{C}=98,9)$ y de FeSi (\% en masa, $\mathrm{Si}=74,6 ; \mathrm{Ca}=0,3 ; \mathrm{Al}=0,7$ y $\mathrm{Fe}=24,4)$, respectivamente y, en el caso de las fundiciones de matriz perlítica y martensítica, se adicionó FeMn (\% en masa, $\mathrm{Mn}=76,4$ y $\mathrm{Fe}=23,6)$ y $\mathrm{Cu}$ metálico $(\%$ en masa, $\mathrm{Cu}=99,8)$ para ajustar el contenido de estos elementos. Posteriormente, la temperatura del metal base se aumentó hasta los $1490-1500{ }^{\circ} \mathrm{C}$ para ser trasladado a una cuchara con $70 \mathrm{~kg}$ de capacidad máxima y llevar a cabo los tratamientos de esferoidización. El método para realizar estos tratamientos fue la metodología "sándwich". Para ello, en la cámara de reacción de esta cuchara se introdujeron $0,6 \mathrm{~kg}$ de la ferroaleación FeSiMg 511 (\% en masa, $\mathrm{Si}=44,7$; $\mathrm{Mg}=5,6 ; \mathrm{Ca}=1,2 ; \mathrm{TR}$ (Tierras Raras) $=0,7 \mathrm{y}$ $\mathrm{Fe}=47,8)$ antes del vertido de los $50 \mathrm{~kg}$ de metal base en su interior. El material cubriente utilizado estaba constituido por recortes de acero $(5-15 \mathrm{~mm})$ procedentes de procesos de estampación. Al finalizar los tratamientos con magnesio, el metal se desescorió en la propia cuchara, se obtuvo una medalla para analizar su composición química y, a continuación, se colaron los moldes. Las piezas rectangulares se fabricaron utilizando moldes de arena aglomerada químicamente. El proceso de inoculación del metal se realizó posicionando un lingote de inoculante (\% en masa, $\mathrm{Si}=70-76 ; \mathrm{Al}=3,1-4,3 ; \mathrm{Ca}=0,3-1,3 \mathrm{y}$ $\mathrm{TR}=0,4-0,5)$ en la copa de colada del molde y justo antes del filtro situado en la parte inferior de dicha copa. En todos los casos, la cantidad de inoculante utilizado fue el $0,20 \%$ del peso de metal colado en el molde.

Tras el enfriamiento de los moldes, las piezas se separaron y, posteriormente, se granallaron. En el caso de la fundición esferoidal ferrítica, las dos piezas se marcaron como "FGE-F" y se sometieron a un tratamiento térmico de recocido (calentamiento hasta $920{ }^{\circ} \mathrm{C}$, mantenimiento durante 1 hora $\mathrm{y}$ enfriamiento a $1^{\circ} \mathrm{C} \mathrm{min}^{-1}$ hasta alcanzar los $300^{\circ} \mathrm{C}$ ). Con el fin de obtener la fundición esferoidal martensítica, las piezas marcadas como "FGE-M" se calentaron hasta alcanzar los $920^{\circ} \mathrm{C}$ y, tras 1 hora de permanencia a esa temperatura, se templaron con agitación en un baño de agua a $20^{\circ} \mathrm{C}$. Las dos piezas fabricadas con fundición esferoidal perlítica (FGE-P) no se sometieron a tratamiento térmico alguno. Una de las dos piezas fabricadas con cada material se cortó con el fin de obtener una probeta metalográfica en la zona central y determinar el índice de esferoidización (IE), la densidad de nódulos (N) y la composición de la matriz metálica. Los valores de estos parámetros se obtuvieron analizando cinco campos de observación diferentes en cada muestra y comparándolos con patrones estándar (Ryntz, 1974; O’Brien et al., 1974). En la preparación de la fundición laminar, FG-PL, se siguió una metodología similar a la descrita anteriormente, prescindiendo del tratamiento de esferoidización. De este modo, tras el ajuste de la composición química del metal fundido en el horno de fusión, éste se trasvasó a la cuchara para colar los moldes posteriormente.

Los contenidos de $\mathrm{C}$ y S se determinaron mediante técnicas de análisis por combustión (LECO CS 200). En el caso del $\mathrm{Si}$, sus contenidos se determinaron

Tabla 1. Composición química de los diferentes tipos de fundición esferoidal utilizados para fabricar las piezas objeto de estudio (\% en masa)

\begin{tabular}{llccccccc}
\hline & & \multicolumn{7}{c}{ Composición química (\%) } \\
\cline { 3 - 9 } Fundición & \multicolumn{1}{c}{ Estructura } & $\mathbf{C}$ & $\mathbf{S i}$ & $\mathbf{M n}$ & $\mathbf{P}$ & $\mathbf{S}$ & $\mathbf{M g}$ & $\mathbf{C u}$ \\
\hline FGE-F & Esferoidal Ferrítica & 3,83 & 2,28 & 0,19 & 0,012 & 0,006 & 0,033 & 0,03 \\
FGE-P & Esferoidal Perlítica & 3,82 & 2,31 & 0,68 & 0,011 & 0,008 & 0,041 & 0,78 \\
FGE-M & Esferoidal Martensítica & 3,82 & 2,31 & 0,68 & 0,011 & 0,008 & 0,041 & 0,78 \\
FG-PL & Laminar Perlítica & 3,68 & 1,80 & 0,54 & 0,016 & 0,034 & $<0,005$ & 0,41 \\
FGE-Si2 & Esferoidal Ferrítica & 3,80 & 1,91 & 0,19 & 0,015 & 0,007 & 0,045 & 0,04 \\
FGE-Si3 & Esferoidal Ferrítica & 3,42 & 2,98 & 0,20 & 0,016 & 0,008 & 0,040 & 0,05 \\
FGE-Si4 & Esferoidal Ferrítica & 3,16 & 4,02 & 0,21 & 0,016 & 0,008 & 0,054 & 0,05 \\
\hline
\end{tabular}


mediante gravimetría y, para el resto de elementos, se utilizó la espectrometría de emisión con plasma acoplado inductivamente (ICP-OES Perkin Elmer Optima 5300).

Para su caracterización microestructural, las probetas extraídas de cada pieza fueron pulidas a espejo y atacadas con el reactivo ácido NITAL 5 (ácido nítrico al 5\% en etanol).

En una segunda etapa y siguiendo la metodología descrita anteriormente, se fabricaron otras tres fundiciones esferoidales, modificadas con diferentes contenidos de $\mathrm{Si}$ (entre 1,91 y 4,02\% en masa, ver Tabla 1), con el fin de estudiar el efecto de este elemento sobre el comportamiento frente a la corrosión. Para obtener estructuras matriciales completamente ferríticas, en estos casos, las piezas fabricadas con las aleaciones FGE-Si2 y FGE-Si3 se sometieron a un tratamiento térmico de recocido similar al aplicado sobre las piezas FGE-F.

Los ensayos electroquímicos se realizaron en una solución de $\mathrm{NaCl} 0,03 \mathrm{M}$ a temperatura ambiente, mediante el empleo de un potenciostato Gamry modelo Reference 600. La celda electroquímica estaba constituida por tres electrodos, uno de referencia de $\mathrm{Ag} / \mathrm{AgCl}(3 \mathrm{M} \mathrm{KCl})$, un contraelectrodo de Pt, y el electrodo de trabajo que estaba constituido por las muestras objeto de estudio. El área expuesta de las muestras es de $1,2 \mathrm{~cm}^{2}$. Previamente a la realización del ensayo, las muestras se rectificaron dejando un acabado superficial con papel de $\mathrm{SiC}$ con una granulometría de 1200 grit. Posteriormente se limpiaron y desengrasaron con alcohol en un baño de ultrasonidos durante 5 minutos.

Las curvas potenciodinámicas se realizaron, aplicando un escalón de potencial, en sentido catódico, de $-0,3 \mathrm{~V}$ respecto al potencial de corrosión (Ecorr). A continuación, se inicia el barrido en sentido anódico a una velocidad de $167 \mu \mathrm{V} \mathrm{s}^{-1}$ hasta alcanzar un valor límite de $0,25 \mathrm{~mA} \mathrm{~cm}^{-2}$, instante en el que se inicia el barrido reverso hasta llegar al potencial inicial.

Las medidas de espectroscopía de impedancia electroquímica se hicieron aplicando una señal sinusoidal de $5 \mathrm{mV}$ de amplitud en un intervalo de frecuencias de $100 \mathrm{kHz}$ a $10 \mathrm{mHz}$ y tomando 10 puntos por década. Se hicieron medidas periódicas cada 24 horas hasta un total de 168 horas de inmersión.

Previa a la realización de las medidas de impedancia se registró la evolución del potencial de circuito abierto (OCP) para las diferentes fundiciones evaluadas durante 10 minutos.

\section{RESULTADOS Y DISCUSIÓN}

Las microestructuras de las fundiciones de hierro no modificadas son las que se presentan en la Figura 1. Se trata de tres fundiciones esferoidales con estructura matricial completamente ferrítica (Fig. 1a), un $90-95 \%$ de perlita y $5-10 \%$ de ferrita (Fig. 1b) y martensítica con el 3-5\% de austenita retenida (Fig. 1c). En todos los casos, los valores del parámetro IE son superiores al $90 \%$ y la densidad de esferoides se encuentra en el intervalo $150-215 \mathrm{~mm}^{-2}$. La fundición laminar perlítica está compuesta por láminas de grafito en una matriz perlítica, la cual contiene algunas trazas de ferrita, normalmente asociadas a las láminas grafíticas (Fig. 1d).

Tras la aplicación del tratamiento térmico para ferritizar la matriz metálica, las piezas fabricadas con las aleaciones FGE-Si2 y FGE-Si3 muestran características estructurales similares a la indicada en la Figura 1a, para la aleación FGE-F. En el caso de la aleación FGE-Si4, la matriz metálica es completamente ferrítica en estado bruto de colada. En los tres casos, los valores del IE son superiores al $90 \%$, aunque la densidad de esferoides aumenta con el contenido de Si en la aleación (209-234 $\mathrm{mm}^{-2}$ para FGE-Si2, 269-305 $\mathrm{mm}^{-2}$ para FGE-Si3 y $311-351 \mathrm{~mm}^{-2}$ para FGE-Si4). En ninguno de los casos estudiados se ha detectado presencia de carburos.

Las medidas del potencial de circuito abierto para las cuatro fundiciones no modificadas muestran la misma tendencia en todos los casos, estabilizándose tras las 5 primeras horas de ensayo y permaneciendo constante a lo largo de toda la experimentación (Fig. 2a). Los valores del potencial para los distintos materiales ensayados oscilan entre -660 y $-710 \mathrm{mV}$. Por otra parte, las fundiciones modificadas (Fig. 2b), presentan unas variaciones muy similares a las descritas anteriormente.

Las curvas de polarización (Fig. 3), indican que nos encontramos ante unos materiales muy activos, en los que el proceso catódico controla el mecanismo de corrosión; es decir, se establece un control por difusión caracterizado por una densidad límite de corriente. En la Tabla 2 se recogen los valores de las densidades de corriente límite $\left(\mathrm{i}_{\mathrm{lim}}\right)$ y velocidades de corrosión (V) que de estos valores se derivan aplicando la Ley de Faraday para las distintas fundiciones estudiadas. Las densidades de corriente límite para las fundiciones no modificadas son del orden de $2,8 \cdot 10^{-5} \mathrm{~A} \mathrm{~cm}^{-2}$ (Fig. 3a). Las fundiciones modificadas presentan un trazado prácticamente similar entre todas ellas y parecido al de los materiales no modificados, excepto para la fundición con el mayor contenido de Si, muestra FGE-Si4, que presenta valores de la densidad de corriente límite ligeramente más bajos, aproximadamente $5,8 \cdot 10^{-6} \mathrm{~A} \mathrm{~cm}^{-2}$ (Fig. 3b). De acuerdo con la ley de Faraday, siendo la reacción principal el paso de $\mathrm{Fe}^{0}$ a $\mathrm{Fe}^{2+}$ y suponiendo un proceso de corrosión uniforme, estas velocidades equivaldrían a una pérdida de masa de $0,029 \mathrm{mg} / \mathrm{cm}^{2} \mathrm{~h}$ $(0,32 \mathrm{~mm} / \mathrm{año})$ y de $0,006 \mathrm{mg} / \mathrm{cm}^{2} \mathrm{~h}(0,068 \mathrm{~mm} / \mathrm{año})$ respectivamente. Desde el punto de vista de corrosión, el Si es uno de los elementos más importantes para aumentar la resistencia a la corrosión de las fundiciones base Fe. Cuando el contenido de silicio en la aleación es menor del 3\%, no se considera que tenga un efecto apreciable sobre la resistencia 
a)

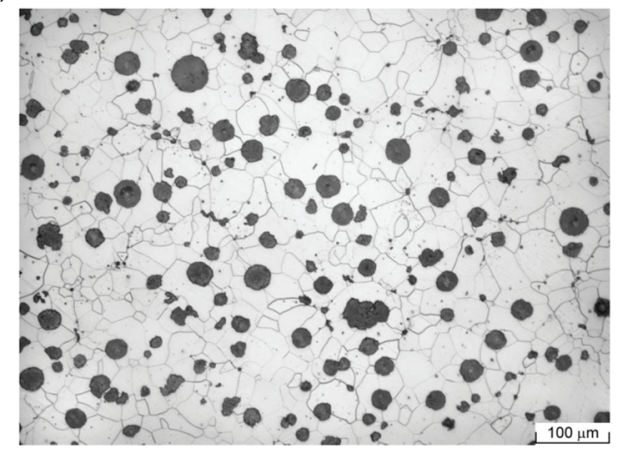

b)

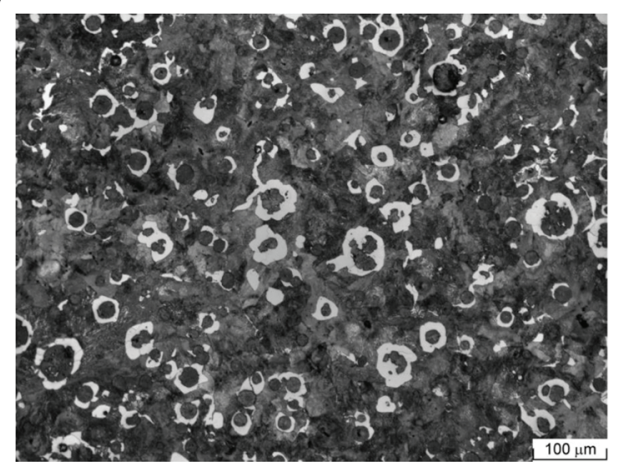

c)

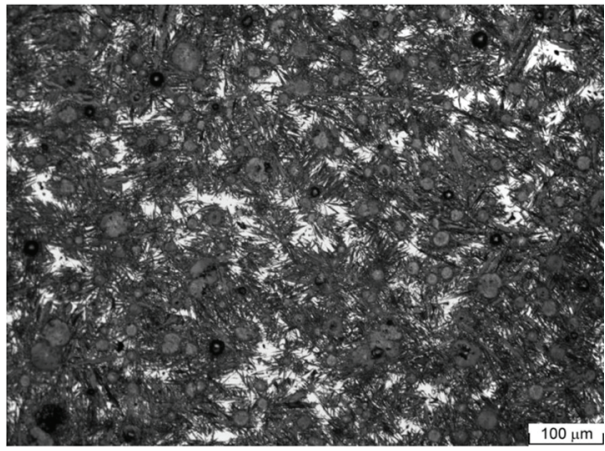

d)

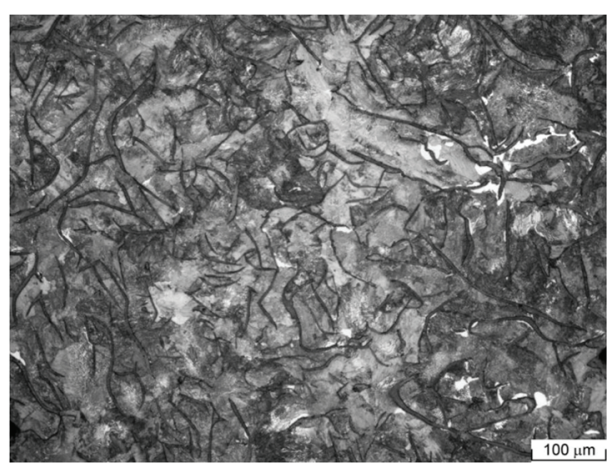

FIGURA 1. Microestructura de las fundiciones de hierro no modificadas: a) fundición esferoidal con estructura matricial completamente ferrítica; b) fundición esferoidal con estructura matricial 90-95\% de perlita y 5-10\% de ferrita;

c) fundiciones esferoidales con estructura martensítica con el 3-5\% de austenita retenida y d) fundición laminar perlítica con trazas de ferrita, normalmente asociadas a las láminas grafíticas.

a la corrosión, tal y como se aprecia también en los resultados aquí encontrados. Cuando el contenido está comprendido entre el 3 y el 10\%, mejora ligeramente su resistencia a la corrosión. La razón de esta mejora (y sobre todo la que se produce cuando el aumento es mayor del 14\%) se debe a la formación de una fina capa de pasivación formada por óxidos hidratados de silicio sobre la superficie del metal. Esta película se forma como consecuencia de la disolución del Fe de la matriz, que deja el silicio expuesto a la disolución (Reynaud, 2010).

En este sentido, podríamos pensar en una mejora relativa desde el punto de vista de resistencia a la corrosión. Sin embargo, esta situación no implica una resistencia frente a la corrosión a largo plazo.

El proceso de corrosión que exhiben estos materiales no es uniforme debido a que la presencia de nódulos de grafito propicia la presencia de pares galvánicos en superficie. El grafito es un material inerte desde el punto de vista de la corrosión y es catódico respecto al hierro. Como resultado, se produce un rápido ataque incluso en medios de corrosividad baja o moderada. Cuando este ataque se produce, se forman capas de óxido (en cuya composición están presentes el Fe, partículas de grafito, aniones presentes en el medio, otros cationes metálicos, etc.) sobre la superficie del material. Si esas capas son compactas y adherentes se reducirá el ataque del medio al metal. Por el contrario, si son porosas y poco adherentes, el proceso continuará aunque con una mayor dificultad.

Como ejemplo representativo de lo que se acaba de explicar se presenta el ataque sufrido por la fundición con estructura ferrítica (Fig. 4). En ella se pueden apreciar los huecos donde había grafito y que se han desprendido debido al ataque corrosivo que se ha producido alrededor de dichos nódulos. En la misma foto puede apreciarse que otros nódulos de mayor tamaño que los anteriores, no se han desprendido todavía. El hierro que se disuelve forma sobre la superficie un óxido muy voluminoso y de carácter no protector.

En la Figura 5a se presentan los diagramas de impedancia electroquímica obtenidos para la fundición esferoidal ferrítica FGE-F, cuyo comportamiento es representativo de las fundiciones sin modificar estudiadas. En la Figura 5b se muestran los espectros de impedancia de la fundición modificada FGE-Si4, que mostraba la menor densidad de corriente de corrosión en las curvas de polarización. Como puede observarse en dicha Figura 5, las fundiciones estudiadas presentan espectros de impedancia similares. En ambos casos los diagramas de impedancia están constituidos por dos constantes de tiempo, localizadas en alta y baja frecuencia, 

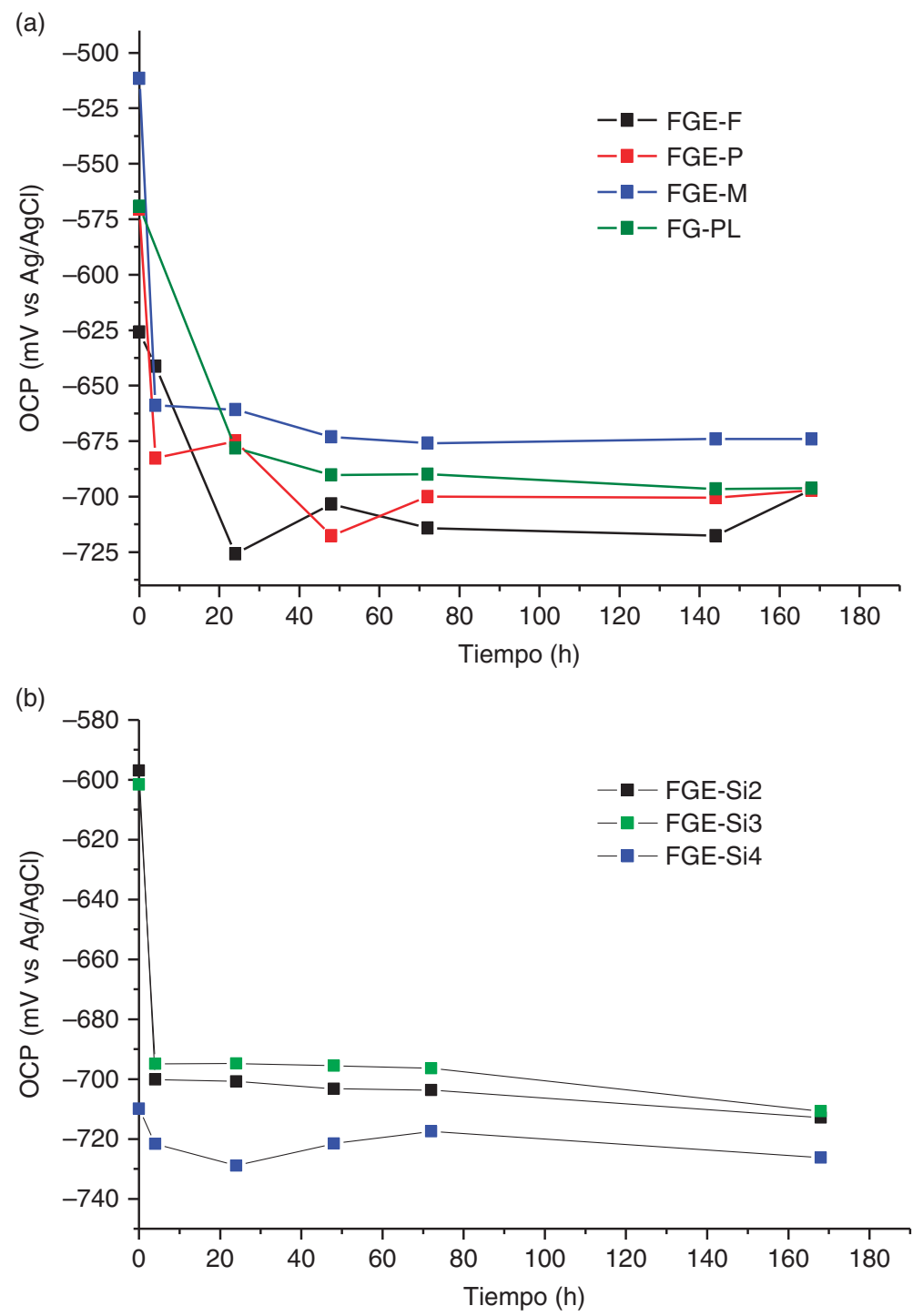

FIGURA 2. Variación del potencial de circuito abierto en: a) las cuatro fundiciones no modificadas y b) las fundiciones modificadas con Si.

respectivamente. La constante de tiempo de alta frecuencia, centrada en $10^{5} \mathrm{~Hz}$, aparece a las 48 horas en las fundiciones no modificadas, mientras que su presencia se distingue desde las 4 horas de inmersión en la aleación modificada. La aparición de esta constante de tiempo se relaciona con la rápida generación de los óxidos de hierro que cubren la superficie de las muestras y que son muy voluminosos y poco adherentes. Estos óxidos se depositan sobre la superficie pero no forman una película compacta que proteja al material, sino que forman una capa porosa que no impide que el proceso corrosivo continúe. Este hecho se asociaría con la aparición de la constante de tiempo descrita a bajas frecuencias, cuyo ángulo de fase alcanza su máximo inicialmente a una frecuencia del orden de $10 \mathrm{~Hz}$ en la fundición esferoidal no modificada. Con el tiempo de inmersión, este máximo se desplaza a frecuencias cada vez más bajas y de forma simultánea el valor del ángulo de fase correspondiente también disminuye, lo que indica que el material base continúa atacándose y exponiendo nueva superficie al medio agresivo.

Por otra parte, y aunque los diagramas de impedancia descritos para la fundición modificada son análogos a las de las no modificadas, es posible distinguir algunas pequeñas diferencias. En el intervalo de bajas frecuencias, la fundición modificada presenta un espectro más estable en el tiempo. Es decir, la constante de tiempo de baja frecuencia aparece centrada en $0,5 \mathrm{~Hz}$ y se mantiene invariante durante todo el tiempo de ensayo.

En general, valores del ángulo de fase tan bajos como los encontrados en el diagrama de Bode, del orden de $40-50^{\circ}$, en frecuencias medias-bajas están relacionados con procesos de difusión. Esto se confirma con el valor de la pendiente, de $-0,55$ a $-0,60$, 
(a)

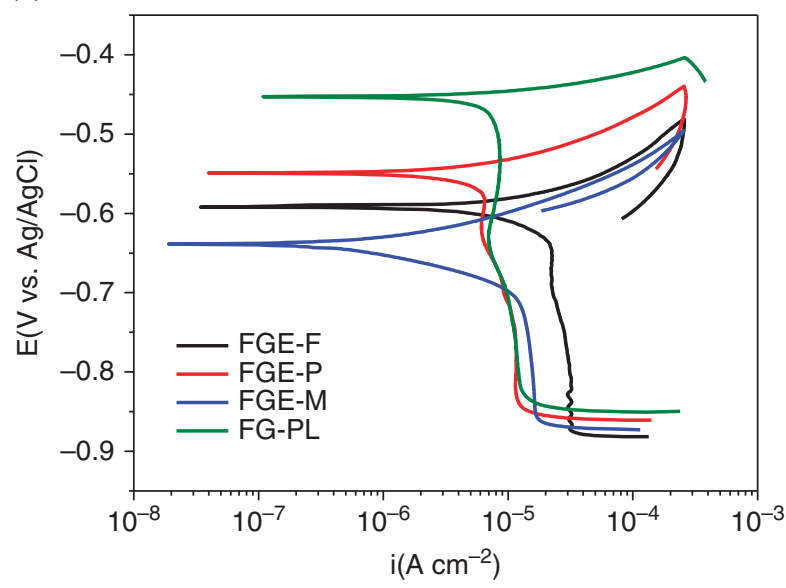

(b)

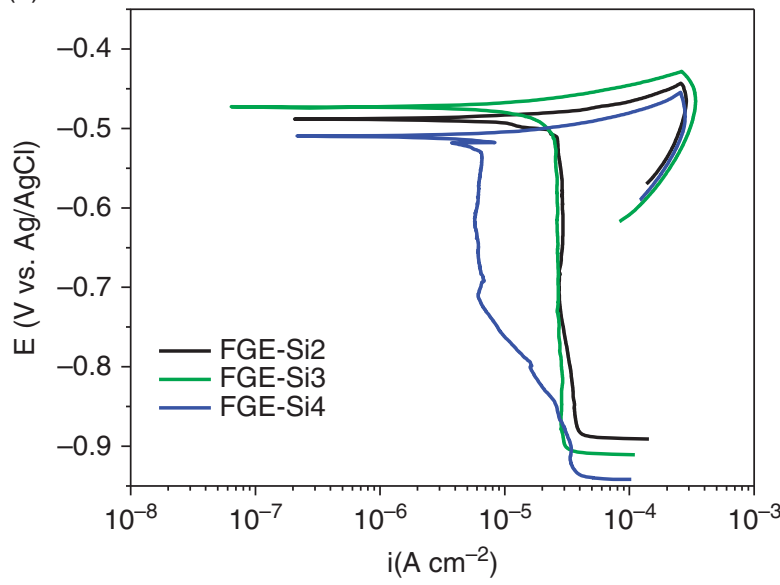

Figura 3. Curvas de polarización obtenidas en: a) las cuatro fundiciones no modificadas y b) las fundiciones modificadas con $\mathrm{Si}$. Todas muestran unos materiales activos cuyo proceso catódico controla el mecanismo de corrosión.

del tramo rectilíneo descrito en el diagrama de Bode del módulo de la impedancia en el mismo intervalo de frecuencias. Por otra parte, su representación en el diagrama de Nysquist se corresponde con un semicírculo muy deprimido. En este caso, además, es

Tabla 2. Densidades de corriente límite y velocidades de corrosión (extrapoladas de dichas densidades) para los distintos tipos de fundición estudiados

\begin{tabular}{lcc}
\hline Fundición & $\begin{array}{c}\mathbf{i}_{\text {lim }} \\
\left(\mathbf{A ~ c m} \mathbf{~ c m}^{-2}\right.\end{array}$ & $\begin{array}{c}\mathbf{V} \\
(\mathbf{m m} / \mathbf{a n ̃ o})\end{array}$ \\
\hline FGE-F & $2,83 \cdot 10^{-5}$ & 0,32 \\
FGE-P & $1,03 \cdot 10^{-5}$ & 0,12 \\
FGE-M & $1,43 \cdot 10^{-5}$ & 0,16 \\
FG-PL & $1,09 \cdot 10^{-5}$ & 0,13 \\
FGE-Si2 & $2,62 \cdot 10^{-5}$ & 0,30 \\
FGE-Si3 & $2,89 \cdot 10^{-5}$ & 0,33 \\
FGE-Si4 & $5,82 \cdot 10^{-6}$ & 0,068 \\
\hline
\end{tabular}

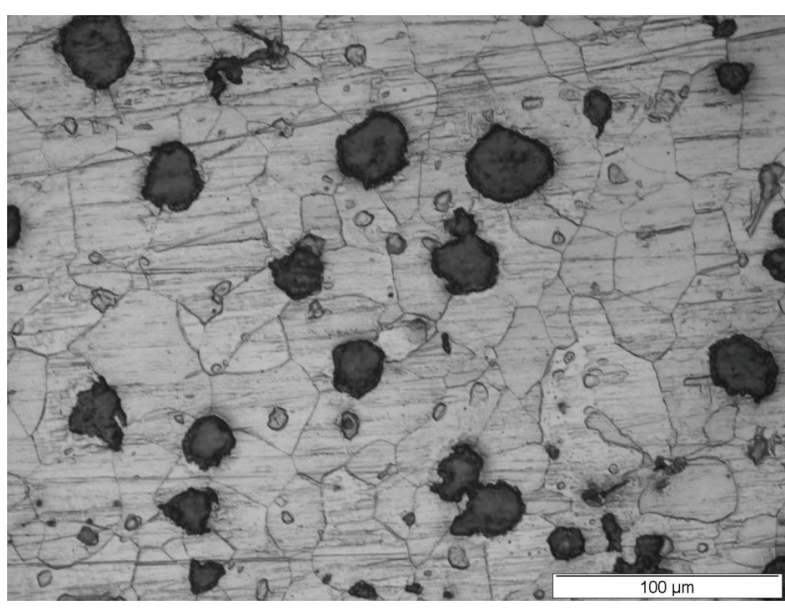

Figura 4. Ataque sufrido por la fundición con estructura matricial completamente ferrítica. Se observan huecos en los que inicialmente existían partículas de grafito.

posible distinguir la existencia de un bucle inductivo en las frecuencias más bajas en los periodos iniciales de inmersión. La aparición de este bucle se relaciona con la adsorción de especies intermedias cloruradas en la intercara metal/solución que intervienen en el proceso de disolución del hierro y que son altamente solubles. De ahí que tenga lugar la desaparición del bucle en periodos de inmersión más largos, a partir de las 72 horas.

Los valores obtenidos experimentalmente de la capacidad asociada a este semicírculo deprimido son muy elevados, del orden de $0,1-5,0 \mathrm{mF} \mathrm{cm}^{-2}$, corroborando que la respuesta del material está dominada por un proceso difusivo, el cual tiene lugar a través de la capa de óxido de hierro voluminosa, poco adherente y defectuosa que se forma sobre la fundición al estar expuesta al medio agresivo.

En procesos electroquímicos como los que suceden en las fundiciones evaluadas, en las que llevan asociados no sólo procesos de transferencia de carga sino también procesos de transporte de masa por difusión, la resistencia que contempla el proceso de difusión se denomina impedancia de Warburg, $\mathrm{Z}_{\mathrm{w}} \mathrm{y}$ está asociada a los fenómenos de la variación de concentración de los reactantes o de los productos o de ambos y se expresa como se indica en la Ecuación (1):

$$
Z_{w}=\frac{\sigma}{\sqrt{w}}-j \frac{\sigma}{\sqrt{w}}
$$

donde $\sigma$ es el coeficiente de difusión de Warburg y está relacionado con las concentraciones y coeficientes de difusión de las especies oxidadas y reducidas. Dicho coeficiente puede calcularse a partir de la expresión descrita por Walter (1986), Ecuación (2):

$$
\sigma=Z i \sqrt{\pi}
$$


(a)

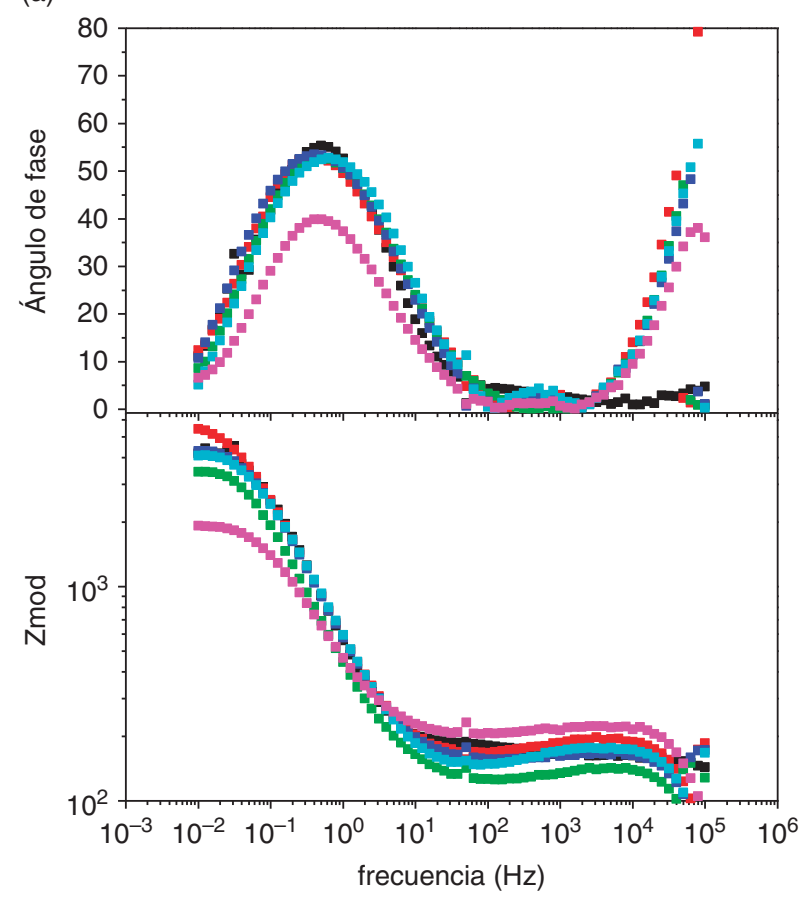

(b)

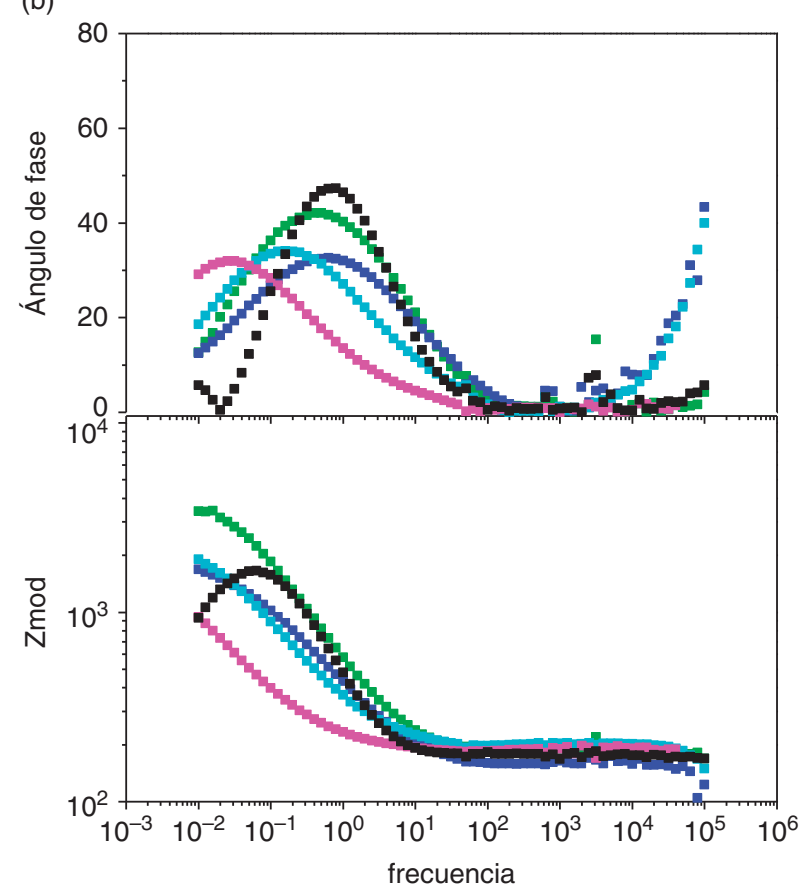

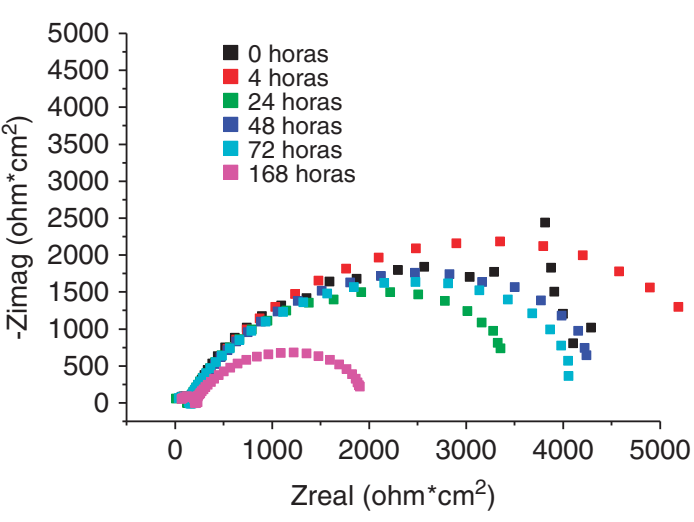

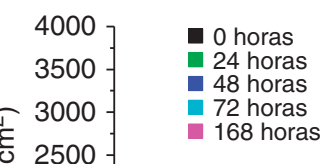

등 2000 -

응 1500

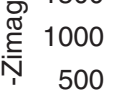

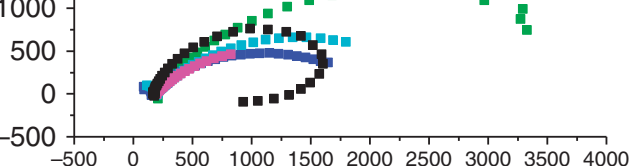

Zreal $\left(\mathrm{ohm}^{*} \mathrm{~cm}^{2}\right)$

FIgURA 5. Evolución temporal de los diagramas de impedancia electroquímica: a) fundición esferoidal ferrítica y b) fundición modificada FGE-Si4, que mostraba la menor densidad de corriente de corrosión en las curvas de polarización.

donde $\mathrm{Zi}$ es el módulo de la impedancia determinado a partir del diagrama de Bode del módulo de la impedancia para una valor de $\mathrm{f}=1 \mathrm{~Hz}$.

Los coeficientes de difusión de la impedancia de Warburg obtenidos para las cuatro fundiciones esferoidales no modificadas son similares. En la Tabla 3 se recogen los valores para la FGE-F como representativa de las no modificadas y de la FGE-Si4 por su mejor comportamiento. En el primer caso, este coeficiente disminuye desde $854 \Omega \cdot \mathrm{s}^{-0,5}$ para 
TABLA 3. Valores obtenidos de coeficientes de difusión de la impedancia de Warburg para las fundiciones esferoidales ferríticas y con un $4 \%$ de $\mathrm{Si}$

\begin{tabular}{lcccc}
\hline & \multicolumn{4}{c}{$\begin{array}{c}\sigma \\
\left(\Omega \cdot \mathbf{s}^{-0,5}\right)\end{array}$} \\
\cline { 2 - 5 } Fundición & $\mathbf{2 4} \mathbf{h}$ & $\mathbf{4 8} \mathbf{h}$ & $\mathbf{7 2} \mathbf{~ h}$ & $\mathbf{1 6 8} \mathbf{h}$ \\
\hline FGE-F & 854,0 & 637,8 & 539,2 & 344,7 \\
FGE-Si4 & 855,2 & 866,6 & 879,3 & 686,7 \\
\hline
\end{tabular}

las 24 horas de inmersión, hasta $344,7 \Omega \cdot \mathrm{s}^{-0,5}$ a las 168 horas. Por el contrario, la fundición modificada FGE-Si4 presenta un coeficiente de difusión que se mantiene superior (en torno a los $800 \Omega \cdot \mathrm{s}^{-0,5}$ ) al de las fundiciones no modificadas, durante todo el período de inmersión evaluado. Este valor sugiere un impedimento para la reacción catódica mayor que el descrito en las fundiciones convencionales. No obstante, estas diferencias no pueden considerarse significativas desde el punto de vista de la cinética corrosiva, corroborándose así los resultados descritos en las curvas de polarización, que indicaban tan solo una ligera disminución de la cinética corrosiva respecto de las fundiciones convencionales evaluadas.

\section{CONCLUSIONES}

El comportamiento frente a la corrosión en un medio de agresividad moderada muestra que todas las fundiciones estudiadas presentan un proceso corrosivo bajo control por difusión, con densidades de corriente de corrosión comprendidas entre 5,8 y $28,0 \mu \mathrm{A} \mathrm{cm}^{-2}$. Este comportamiento es comparable en las cuatro fundiciones de hierro no modificadas, por lo que no muestra dependencia alguna de la morfología del grafito (láminas o esferoides) y/o de los constituyentes presentes en la matriz metálica (ferrita, perlita o martensita).

En relación a las fundiciones esferoidales modificadas con silicio, la aleación con el mayor contenido de silicio presenta una ligera mejora del comportamiento frente a la corrosión respecto de las fundiciones convencionales y también en relación a aquellas aleaciones modificadas con menores contenidos de este elemento. No obstante, los resultados sugieren que este contenido de $\mathrm{Si}$ es aún insuficiente para obtener unas propiedades óptimas de resistencia a la corrosión.

En cuanto a la morfología del ataque desarrollado, se trata de un proceso de corrosión uniforme, con la formación de una capa voluminosa de óxido poco adherente sobre la superficie de la aleación. No obstante, la presencia del grafito da lugar a la aparición de micropilas galvánicas de corrosión que producen un ataque localizado con descolgamiento del grafito, como resultado del ataque de la intercara entre los nódulos de grafito y la matriz metálica.

\section{AGRADECIMIENTOS}

Los autores agradecen la financiación obtenida por el Centro de Investigación Cooperativa CIC Margune (proyecto ETORTEK 2010-2012) y la ayuda prestada por los responsables de TQC Technologies, S.L.U. en la ejecución de las pruebas experimentales.

\section{REFERENCIAS}

Camba, C., Varela, A., Blázquez, V., García, A., Mier, J.L., Barbadillo, F. (2010). Comportamiento al desgaste de fundiciones tipo "silal". Rev. Metal. 46 (N. Extr.), 40-46. http://dx.doi.org/10.3989/revmetalmadrid.04XIIPMS.

DeBerry, D.W., Kidwell, J.R., Malish, D.A. (1982). Corrosion in potable water systems. Final Report $\mathrm{N}^{\circ} 68-01-5834$, United States Environmental Protection Agency, USA.

de la Torre, U., Loizaga, A., Lacaze, J., Sertucha, J. (2014). As cast high silicon ductile irons with optimised mechanical properties and remarkable fatigue properties. Mater. Sci. Technol. 30 (12), 1425-1431. http://dx.doi.org/10.1179/174 3284713 Y.0000000483.

Morcillo, M., de la Fuente, D., Díaz, I., Cano, H. (2011). Atmospheric corrosion of mild Steel. Rev. Metal. 47 (5) 426-444. http://dx.doi.org/10.3989/revmetalm.1125.

O'Brien, J., Adams, A., Avedisian, A., Barnes, G.J., Booth, BN., Dubberstein, G., House, W., Janowak, J.F., Langnerm E.E., Lansing, J.H., Lee, RS., Nelson, C.D., Parks, TW., Peacock, J.H., Thomson, RS., Vanik, J.S., Warrick. R.J. (1974). Reference microstructure for measurement of pearlite and ferrite content in ductile iron microstructures. AFS Report. AFS Transactions 82, 545-550.

Reynaud, A. (2010). Corrosion of Cast Irons, in Shreir's Corrosion, T.J.A. Richardson, Ed. Vol. 3, Cap. 2, Elsevier, Amsterdam, The Netherlands, pp.1737-1788.

Ryntz, E.F. (1974). Reference microstructures for visual estimation of iron carbide content in nodular iron. AFS Transactions 82, 551-554

Sun, Y., Hu, S., Xiao, Z., You, S., Zhao J., Lv, Y. (2012). Effects of nickel on low-temperature impact toughness and corrosion resistance of high-ductility ductile iron. Mater. Des. 41 (1), 37-42. http://dx.doi.org/10.1016/j.matdes.2012.03.039.

Tiedje, N.S. (2010). Solidification, processing and properties of ductile cast iron. Mater. Sci. Technol. 26, 505-514. http:// dx.doi.org/10.1179/026708310X12668415533649.

Tullmin, M., Roberge, P.R. (2000). Atmospheric Corrosion, in Uhlig's Corrosion Handbook, Cap. 18, Ed. John Wiley \& Sons.

Walter, G.G.W. (1986). A review of impedance plot methods used for corrosion performance analysis of painted metals. Corros. Sci. 26 (9), 681-703

Zhou, Y., Lu, Z., Zhan, M. (2007). An investigation of the erosion-corrosion characteristics of ductile cast iron. Mater. Des. 28 (1), 260-265. http://dx.doi.org/10.1016/j. matdes.2005.07.011. 